\title{
INTERLEUKIN-6 DERIVED FROM HYPOXIC MYOCYTES PROMOTES NEUTROPHIL-MEDIATED REPERFUSION INJURY IN MYOCARDIUM
}

Yoshiki Sawa, MD

Hajime Ichikawa, MD

Koji Kagisaki, MD

Toshihiro Ohata, MD

Hikaru Matsuda, MD
Background: Reperfusion injury in the myocardium has recently been considered to be a type of inflammation, and close attention has been paid to the possible involvement of neutrophils, complement, and cytokines in the onset of this injury. Recently, it has been reported that serum levels of interleukin-6 are elevated significantly after myocardial infarction. The major site of interleukin-6 production and its exact roles are still unknown. In this study, we hypothesized that myocytes may produce interleukin- 6 during hypoxia and this may play a role in neutrophil-mediated reperfusion injury. Methods and results: In the clinical study, 20 patients who underwent coronary artery bypass grafting were divided into 2 groups: group F, in which patients were treated with a serine protease inhibitor (FUT-175, $2 \mathrm{mg} / \mathrm{kg}$ per hour) during cardiopulmonary bypass, and group $\mathrm{C}$ (untreated patients). In group $\mathrm{C}$, myocardial interleukin- 6 production, as determined by the difference between the interleukin-6 level in the cardiopulmonary bypass circuit and its level in coronary venous blood, increased significantly after reperfusion $(12 \pm 4 \mathrm{pg} / \mathrm{mL})$ as compared with that before aortic crossclamping $(2 \pm 2 \mathrm{pg} / \mathrm{mL})$. In group $F$, the increase in the interleukin-6 level was suppressed significantly (before aortic crossclamping, $3 \pm 2 \mathrm{pg} / \mathrm{mL}$; after reperfusion, $4 \pm 3 \mathrm{pg} / \mathrm{mL}$ ). The interleukin-6 production differed significantly between group $C$ and group $F$. In the in vitro experimental study, the supernatant from myocytes exposed to 2 hours of

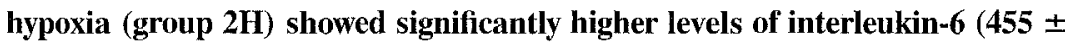
$260 \mathrm{pg} / \mathrm{mL}$ ) than that from normoxic myocytes (group N) $(47 \pm 15 \mathrm{pg} / \mathrm{mL}$ ). This interleukin-6 production was suppressed by the addition of FUT-175 $(123 \pm 24 \mathrm{pg} / \mathrm{mL})$. The interleukin-6 production by endothelial cells of coronary vessels did not differ between group $2 \mathrm{H}(283 \pm 151 \mathrm{pg} / \mathrm{mL})$ and group N $(151 \pm 86 \mathrm{pg} / \mathrm{mL})$. In a coincubation system with a monolayer of endothelial cells on collagen membrane and myocytes under collagen membrane in a modified Boyden chamber, 2 hours of coincubation showed a significantly higher percent of neutrophil transendothelial migration (group $2 \mathrm{H}$ vs $\mathrm{N}, 78 \%$ $\pm 13 \%$ vs $26 \% \pm 11 \%$ ), value of chemiluminescence ( $22 \pm 8$ vs $5 \pm 2 \times 10^{3}$ counts $/ 3$ minutes $)$, and percent of irreversibly damaged myocytes $(48 \% \pm 17 \%$ vs $12 \% \pm 8 \%$ ) than normoxic coincubation. In contrast, anti-interleukin-6 monoclonal antibody significantly attenuated neutrophil transendothelial migration $(42 \% \pm 19 \%)$ and irreversible damage of myocytes $(26 \% \pm 15 \%)$ in 2 hours of coincubation. Conclusions: Interleukin-6 is produced from myocardium during ischemia and reperfusion in patients undergoing coronary bypass grafting. This interleukin-6 may be derived from hypoxic myocytes and play a role in neutrophil-mediated reperfusion injury in myocardium. (J Thorac Cardiovasc Surg 1998;116:511-7)
From the First Department of Surgery, Osaka University Medical School, Osaka, Japan.

Received for publication Dec 29, 1998; revisions requested Feb 23, 1998; revisions received April 30, 1998; accepted for publication May 13, 1998.

\footnotetext{
Address for reprints: Yoshiki Sawa, MD, First Department of Surgery, Osaka University Medical School, 2-2, Yamada-oka, Suita, Osaka 565, Japan.

Copyright (C) 1998 by Mosby, Inc.

$0022-5223 / 98 \$ 5.00+0 \quad \mathbf{1 2 / 1 / 9 1 8 6 2}$
} 
nflammatory response has recently been considlered to be involved in a mechanism of postischemic reperfusion injury in myocardium, and close attention has been paid to the possible involvement of ncutrophils, complement, and cytokines in the onset of this injury. ${ }^{1-6}$ We previously suggested the involvement of neutrophils in this injury, on the grounds that myocardial reperfusion injury during surgery was reduced by reperfusion with leukocytedepleted blood, ${ }^{4-6}$ by the use of cyclic adenosine monophosphate phosphodiesterase inhibitor, which inhibits leukocyte aggregation, ${ }^{7}$ and by the use of $\mathrm{Na}^{+} / \mathrm{H}^{+}$exchanger inhibitors. ${ }^{8}$ We have also reported that ischemic cardiac myocytes produced signaling factors, resulting in enhancement of neutrophil migration into the injured area and aggravation of myocardial injury. ' However, such a myocyte-derived factor that promotes the neutrophilmediated reperfusion injury has not yet been comprehensively studied.

Meanwhile, it has been reported that serum levels of cytokines in blood, especially that of interleukin-6 (IL-6), are elevated significantly after myocardial infarction $^{10,11}$ and that LL-6 messenger RNA (mRNA) is expressed in infarct myocardium ${ }^{12}$ or hypoxic neonatal myocytes. ${ }^{13}$ Thus the possible involvement of IL-6, an inflammation-related cytokine, in myocardial reperfusion injury has been attracting attention. We previously reported that although extracorporeal circulation induces inflammatory reactions and elevates the levels of inflammatory cytokines such as IL-6 and IL-8, these reactions are suppressed by a serine protease inhibitor nafamostat mesylate (FUT-175, Torii Pharmaceutical Company, Tokyo, Japan), possibly leading to reduced myocardial injury. ${ }^{14}$ It therefore seems likely that IL-6 may be produced possibly via inflammatory response including a protease-mediated pathway from myocardium during postischemic reperfusion in patients undergoing coronary artery bypass grafting (CABG) and that it is responsible for exacerbation of neutrophil-mediated myocardial injury. However, the major site of IL-6 production in the human myocardium and its exact roles are still unknown.

We recently carried out a clinical study in which IL-6 levels in coronary venous blood were measured in patients undergoing $C A B G$. At the same time, we performed an experiment in vitro to examine whether IL-6 was produced possibly via a protease-mediated pathway by hypoxic cardiac myocytes and whether this might be responsible for neutrophil-mediated myocardial reperfusion injury.

\section{Patients and methods}

Clinical study. The subjects of the clinical study were 20 patients who underwent CABG in 1994 at the First Department of Surgery, Osaka University Hospital. Informed consent according to the Institutional Review Board in Osaka University was obtained from all patients. Neither the patient nor the surgeon was informed of the group in which that patient was involved, but the investigators were not blinded. Patients were randomly divided into 2 groups: group F (patients treated with FUT-175) and group $\mathrm{C}$ (untreated patients). Conventional cold blood cardioplegic solution $\left(15^{\circ} \mathrm{C}\right)$ containing only potassium $(16 \mathrm{mEq} / \mathrm{L})$ was used for myocardial protection. It was antegradcly administered at a rate of $10 \mathrm{~mL} / \mathrm{kg}$, and then half its volume was administered retrogradely every 20 minutes. Its myocardial temperature was kept below $20^{\circ} \mathrm{C}$. In all patients, membrane oxygenators (Capiox, Terumo, Tokyo, Japan) were used and the temperature of the cardiopulmonary bypass (CPB) circuit was maintained at $34^{\circ} \mathrm{C}$ as a tepid CPB.

In all group $\mathrm{F}$ patients, FUT-175 was administered into the CPB circuit at a rate of $2 \mathrm{mg} / \mathrm{kg}$ per hour during CPB for about 130 minutes (132 \pm 36 minutes). FUT-175 is an inhibitor of serine protease, attenuates the inflammatory response, and has no side effect when used at a dose less than $2 \mathrm{mg} / \mathrm{kg}$ per hour for human beings. ${ }^{14}$ The patients were administered neither anti-inflammatory drugs nor antifibrinolytic drugs. Four patients in group $\mathrm{F}$ and 5 patients in group $C$ received a transfusion after $\mathrm{CPB}$, but no patient received one during $\mathrm{CPB}$.

The mean age was $63 \pm 18$ years in group $C$ and $61 \pm$ 16 years in group $\mathbf{F}$. The number of grafts averaged $2.8 \pm$ 0.4 in group $\mathrm{C}$ and $2.6 \pm 0.6$ in group $\mathrm{F}$. The duration of aortic crossclamping averaged $95 \pm 45$ minutes in group $\mathrm{C}$ and $88 \pm 26$ minutes in group $F$. The duration of CPB averaged $132 \pm 36$ minutes in group $\mathrm{C}$ and $119 \pm 29$ minutes in group F. None of these parameters differed significantly between the 2 groups (Table I). The postoperative course was uneventful in all cases.

In both groups, coronary sinus blood (CS) and arterial blood in the CPB circuit (A) were sampled before aortic crossclamping and 10 minutes after reperfusion. The IL-6 levels of these samples were determined by enzyme-linked immunosorbent assay. ${ }^{14}$ Moreover, CS-A difference of malondialdehyde 10 minutes after reperfusion and serum level of maximum creatine kinase $\mathrm{MB}$ for 24 hours after the operation were also measured and compared between the groups. ${ }^{14}$

In vitro experimental study. An in vitro experiment was carried out with cardiac myocytes and vascular endothelial cells from rats to examine whether IL- 6 is produced by hypoxic myocytes and whether this enhances neutrophil transendothelial migration in association with myocyte damage. Myocytes and endothelial cells were isolated from the hearts of Wistar rats, weighing 150 to $200 \mathrm{~g}$, by means of retrograde irrigation with collagenase and Percoll fractionation. Neutrophils were isolated from the 
Table I. Clinical data in both groups

\begin{tabular}{|c|c|c|c|}
\hline & Untreated group & FUT-175-treated group & P value \\
\hline Age & $63 \pm 18 y$ & $61 \pm 16 y$ & $\mathrm{NS}$ \\
\hline No. of grafts & $2.8 \pm 0.4$ & $2.6 \pm 0.6$ & NS \\
\hline Duration of $\mathrm{AXC}$ & $95 \pm 45 \mathrm{~min}$ & $88 \pm 26 \mathrm{~min}$ & NS \\
\hline Duration of CPB & $132 \pm 36 \mathrm{~min}$ & $119 \pm 29 \min$ & NS \\
\hline CS-A of IL- 6 before AXC & $2 \pm 2 \mathrm{pg} / \mathrm{ml}$ & $3 \pm 2 \mathrm{pg} / \mathrm{ml}$ & NS \\
\hline After reperfusion & $12 \pm 4 \mathrm{pg} / \mathrm{ml}$ & $4 \pm 3 \mathrm{pg} / \mathrm{ml}$ & $P=.04$ \\
\hline CS-A of MDA & $2.1 \pm 0.4 \mathrm{nmol} / \mathrm{ml}$ & $0.7 \pm 0.3 \mathrm{nmol} / \mathrm{ml}$ & $P=.04$ \\
\hline Max CK-MB & $43 \pm 12 \mathrm{IU} / \mathrm{L}$ & $15 \pm 6 \mathrm{IU} / \mathrm{L}$ & $P=.03$ \\
\hline
\end{tabular}

$A X C$, Aortic crossclamp; $C P B$, cardiopulmonary bypass; $C S-A$, coronary sinus-arterial difference; $M D A$, malondialdchyde; $C K-M B$, creatine kinase-MB.

blood of the same rats with the use of Pcrcoll fractionation as described before. ${ }^{9}$

An in vitro experimental model as shown in Fig. 1, across which neutrophils can migratc, was composed of a uniform upper chamber of endothelial cells and a lower chamber of myocardial cells in a modified Boyden chamber. A collagen membrane served as a border between the 2 layers. ${ }^{9}$ By means of this model, only isolated cardiac myocytes in the lower chamber were exposed to 2 hours of hypoxia $(2 \mathrm{H})$ which can cause severe reversible damage to myocardial cells. ${ }^{9}$ After reoxygenation of these myocytes, a monolayer of endothelial cells in the upper chamber was combined with that in the lower chamber. Subsequently, neutrophils, labeled with a nonspccific fluorescent dye (PKH2), were applied to the upper chamber (endothelial cells) of the model, and the fluorescence intensity of the neutrophils that had penetrated into the lower layer (myocardial cells) was measured with a fluorescent spectroscope FR1500 (Shimadzu, Kyoto, Japan) 1 hour after reoxygenation. The proportion of the fluorescence of the neutrophils that had migrated into the lower chamber relative to that of all neutrophils applied was considered as the neutrophil migration rate. At the same time, the fluorescence intensity of neutrophils adhering directly to the myocytes was measured and the proportion relative to the total fluorescence intensity of all neutrophils applied was regarded as the neutrophil adherence rate. Moreover, the proportion of injured myocytes was also measured, by means of trypan blue staining, and the 10-minute cumulative chemiluminescence, generated by injured myocytes, was measured with a Luminometer device (Nichinonn, Tokyo, Japan).

These coincubation models were divided into 3 groups: group $\mathrm{N}$ (normoxia), group $2 \mathrm{H}$ (exposed to 2 hours of hypoxia), and group $2 \mathrm{H}$ with monoclonal antibody antiIL-6, which was exposed to hypoxia and administered anti-IL-6 monoclonal antibody (Genzyme Corporation, Cambridge, Mass) during hypoxia. Hypoxia was induced by consumption of oxygen in a closed gas pack system (Becton Dickinson \& Co, Rutherford, NJ) with palladium as a catalyst. Hypoxia was continued for 2 hours, the hypoxic level being monitored with methylene blue.

IL-6 levels in the supernatant of cultured myocytes or endothelial cells after 2 hours of hypoxia were measured by enzyme-linked immunosorbent assay. ${ }^{15}$ FUT-175 ( $\times 10$ nmol/L) was administerce to myocytes during hypoxia to assess whether protease may play a role in the production of IL-6 from myocytes.
Values of each parameter were expressed as mean \pm standard deviation. The significance of differences within the same group was tested with the use of the paired $t$ test. The unpaired $t$ test was used to test the significance of differences betwcen any two groups. Analysis of variance and subsequent post hoc test were used to test the significance of differences among 3 or more groups.

\section{Results}

\section{Clinical study}

IL-6 production from human myocardium. In the control group, myocardial IL-6 production, as determined by the difference between the IL- 6 level in the arterial blood in the CPB circuit and its level in coronary venous blood, increased significantly $(P=$ .04 ) after reperfusion (postreperfusion, $12 \pm 4$ $\mathrm{pg} / \mathrm{mL}$ ) as compared with that before aortic crossclamping (before crossclamping, $2 \pm 2 \mathrm{pg} / \mathrm{mL}$ ).

In the FUT-475-treated group, the increase in the IL-6 level was suppressed significantly (before crossclamping, $3 \pm 2$; after reperfusion, $4 \pm 3$ ). Thus IL-6 production differed significantly between the control group and the FUT-175-treated group $(P=.04$, Table I, Fig. 2).

Myocardial damage correlated with $I L-6$. In the FUT-175-treated group, the CS-A difference of malondialdehyde was suppressed significantly less than in the control group (FUT, $0.7 \pm 0.3$; control, $2.1 \pm 0.4, P=.04$ ). Maximum serum level of creatine kinase $\mathrm{MB}$ during 24 hours of postoperative course was significantly $(P=.03)$ lower in the FUT-175-treated group $(15 \pm 6)$ than that in the control group $(43 \pm 12)$ (Table I).

\section{In vitro experiment}

Neutrophil migration. The neutrophil migration rate in group $2 \mathrm{H}(78 \% \pm 13 \%)$ was significantly higher than that in group $\mathrm{N}(26 \% \pm 11 \%)(P=.04)$. The increase in this parameter after 2 hours of hypoxia was suppressed significantly by treatment with anti-IL-6 monoclonal antibody $(42 \% \pm 19 \%)$ $(P=0.04$, Fig. 3). 


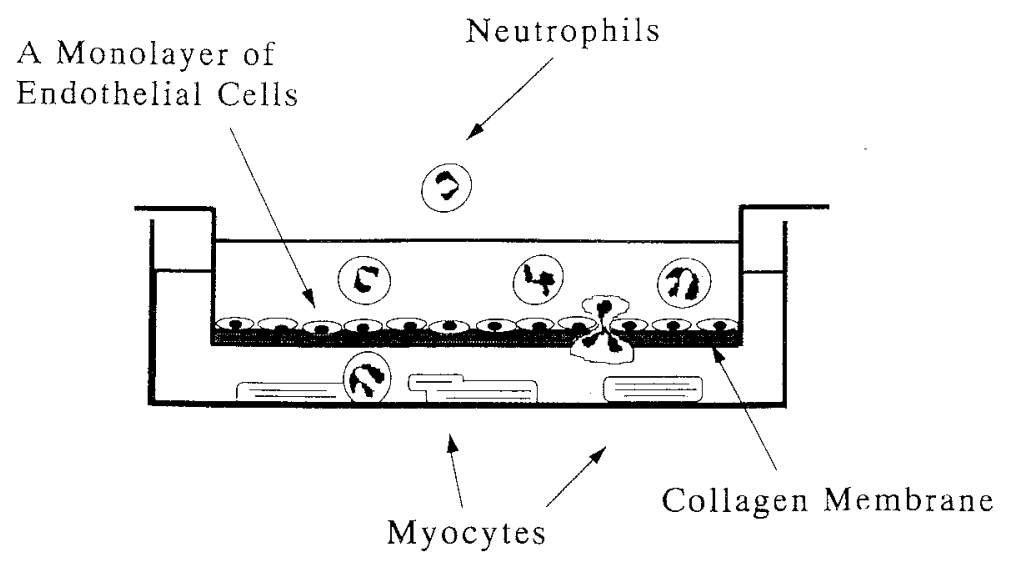

Fig. 1. An in vitro experimental model for neutrophil migration. The model, across which neutrophils can migrate, is composed of a uniform upper layer of endothelial cells and a lower layer of myocytes in a modified Boyden chamber. A collagen membrane serves as a border between the two layers.

Neutrophil adherence. The percentage of neutrophils that adhered to myocytes after penetration across the endothelial cell layer was significantly higher in group $2 \mathrm{H}(64 \% \pm 21 \%)$ than in group $\mathrm{N}$ $(22 \% \pm 12 \%)(P<.05)$. The increase in the neutrophil attachment rate after 2 hours of hypoxia was suppressed significantly by treatment with antiIL-6 monoclonal antibody $(32 \% \pm 19 \%)(P=.04$, Fig. 4).

Myocyte damage. The percentage of damaged myocytes, that is, cells positively stained by trypan blue, after 2 hours of hypoxia $(48 \% \pm 17 \%)$ was significantly higher than that after normoxia $(12 \% \pm$ $8 \%)(P=.04)$. The increase in this parameter after 2 hours of hypoxia was suppressed significantly by treatment with anti-IL-6 monoclonal antibody (26\% $\pm 15 \%)(P=0.04$, Fig. $5, a)$.

The chemiluminescence $\left(\times 10^{3}\right)$, determined simultaneously as an indicator of oxygen-derived free radical production, was significantly higher after 2 hours of hypoxia $(22 \pm 8)$ than after normoxia $(5 \pm$ 2 ), but its increase after 2 hours of hypoxia was suppressed significantly by treatment with anti-IL-6 antibody $(7 \pm 3)(P=.04$, Fig. 5, b).

IL-6 production by myocytes and endothelial cells. The IL-6 production by endothelial cells of coronary vessels did not differ between group $2 \mathrm{H}(283 \pm 151)$ and group $\mathrm{N}(151 \pm 86)(P=.06)$ (Fig. 6, a). The IL-6 level in the supernatant of cultured myocardial cells was significantly higher in group $2 \mathrm{H}(455 \pm 260$ $\mathrm{pg} / \mathrm{mL})$ than in group $\mathrm{N}(47 \pm 15 \mathrm{pg} / \mathrm{mL})(P=.04)$. FUT-175 treatment significantly suppressed IL-6 production in myocardial cells $(123 \pm 24 \mathrm{pg} / \mathrm{mL})$ as compared with that in group $2 \mathrm{H}(P=.04$, Fig. $6, b)$.

\section{Discussion}

We previously reported findings suggesting the involvement of neutrophils in damage to ischemic myocytes, including findings that adhesion molecules are involved in the neutrophil migration into injured myocardium and that the recognition of injured areas of myocardium by circulating neutrophils is promoted by a signaling factor produced by myocytes injured by ischemia. ${ }^{9}$ Our clinical study, presented in this paper, has revealed that during cardiac operations on adult human beings, ischemia resulted in an increase in myocardial IL-6 production and this increase was suppressed by FUT-175, a serine protease inhibitor. Our in vitro study revealed that after 2 hours of hypoxia, the IL-6 production by myocytes increased significantly as compared with IL-6 production by endothelial cells, resulting in enhanced adhesion of neutrophils to the vascular endothelium. And neutrophil migration in association with myocyte damage observed in a co-culture system of myocytes and coronary vascular endothelial cells after 2 hours of hypoxia and this neutrophilmediated myocyte damage was suppressed by antiIL-6 monoclonal antibody. These results indicate that myocytes produce cytokines such as IL-6 when exposed to ischemia and that these cytokines are involved in the enhancement of neutrophil-mediated reperfusion injury in myocardium. The pattern of mRNA for IL-6 in hypoxic neonatal rat myocytes has been reported ${ }^{13}$ before, but there have been no prior reports dealing with direct production and the role of IL- 6 by adult and/or human myocytes.

The results of this study suggest that one possible role of a cytokine whose production by myocytes is 


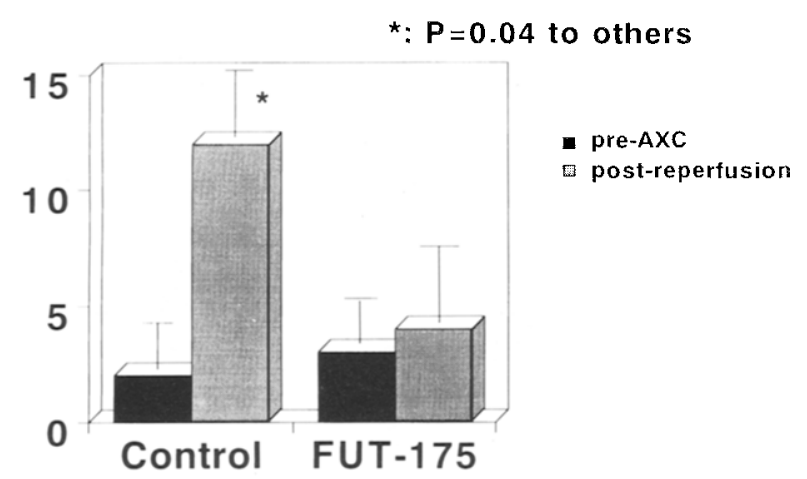

Fig. 2. IL-6 production after postischemic reperfusion during cardiac operations. Myocardial IL -6 production, as determined by the difference between the IL-6 level in the extracorporeal circuit and its level in coronary venous blood, was increased significantly after the release of aortic occlusion in the untreated group. This increase was suppressed significantly in the FUT-175-treated group. IL-6 production differed significantly between the two groups. $A X C$, Aortic crossclamping.

enhanced during ischemia, such as IL-6, is the enhancement of neutrophil-mediated reperfusion injury including neutrophil migration and neutrophil adherence in association with myocyte damage. However, the enhancement of neutrophil migration seems to be multifactorial and is unlikely to be caused by IL- 6 alone, considering that (1) cytokines form a network and are kept in balance with each other ${ }^{15,16}$ and (2) no investigators have reported on intercellular adhesion molecule- 1 expression in vascular endothelial cells induced directly by IL- $6 .{ }^{17} \mathrm{It}$ is therefore plausible to suggest from the results of this study that an increase in IL-6 stimulates the induction of some other chemotactic factor $(\mathrm{eg}$, IL-8), eventually leading to enhancement of neutrophil infiltration, ${ }^{18}$ rather than attributing promotion of neutrophil migration after reperfusion to the direct action of IL-6. ${ }^{17}$

The Baylor group reported that cardiac lymph, collected from the hearts of dogs after postischemic reperfusion, exerted a chemotactic effect, that this lymph contained significant amounts of IL-6, and that the adhesion of neutrophils to the vascular endothelium was suppressed by anti-IL-6 monoclonal antibody. ${ }^{17}$ These findings are identical to those obtained in the present study. It seems likely that the chemotactic factors detected in vivo in cardiac lymph by the Baylor group ${ }^{17}$ are identical to the factors produced directly by ischemic cardiac myocytes in our study, and that these factors passed out

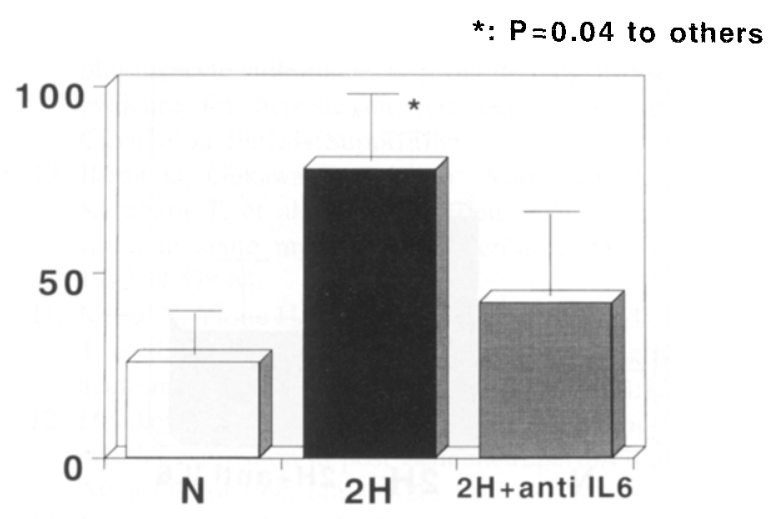

Fig. 3. Neutrophil migration. The neutrophil migration rate was significantly higher in group $2 \mathrm{H}$ (exposed to hypoxia for 2 hours) than in group $N$ (normoxia). The increase in this parameter after 2 hours of hypoxia was suppressed significantly by treatment with anti-IL-6 monoclonal antibody.

of the cells and into coronary veins, as reflected by the detection of IL-6 in coronary venous blood of patients undergoing cardiac operations in the present study.

No detailed study has been carried out concerning the involvement of protease in IL-6 production. It has, however, been reported that protease is involved in the release of cytokines after their production in cytokine-producing cells. ${ }^{19}$ This finding is endorsed by the finding from the present study in vivo and in vitro that IL-6 production was suppressed by a protease inhibitor. We previously reported the possibility that FUT-175 suppresses reperfusion injury of the myocardium, ${ }^{14}$ probably because this protease inhibitor reduced local cytokine production such as IL-6 production in myocardium. FUT-175 is a nonspecific serine protease inhibitor, and there is a complex mechanism of interactions that may take place upstream from IL-6 production. Therefore it is difficult to prove the direct evidence of a protease-dependent pathway of IL-6 production by the present study. However, the present study in vivo and in vitro suggests that inflammatory response including a protease may mediate IL-6 production and further investigations are needed to clarify its mechanism.

In the present study, anti-IL-6 monoclonal antibody in the in vitro experimental study and FUT-175 in the clinical study attenuated neutrophil-mediated reperfusion injury in myocardium. This suggested that inflammatory response including both protease and IL-6 related the neutrophil-mediated myocar- 


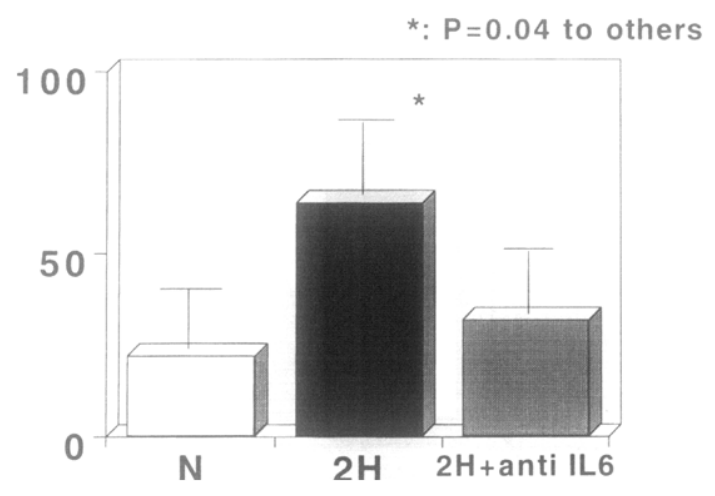

Fig. 4. Neutrophil adherence. The percentage of neutrophils that adhered to myocytes after migration across a monolayer of endothelial cells was significantly higher in group $2 \mathrm{H}$ than in group $\mathrm{N}$. The increase in this parameter after 2 hours of hypoxia was suppressed significantly by treatment with anti-IL-6 monoclonal antibody.

(a) Myocyte damage

(b) Chemiluminescence
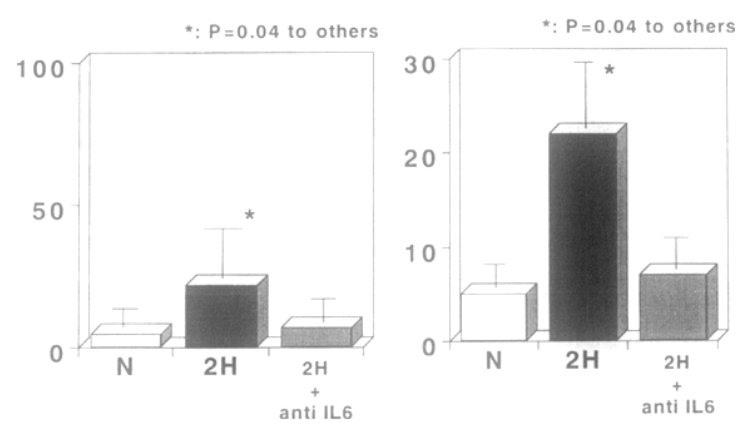

Fig. 5. Percentage of damaged myocytes. a, The percentage of damaged myocytes, that is, myocytes stained positively by trypan blue, was significantly higher in group $2 \mathrm{H}$ than in group $\mathrm{N}$. The increase in this parameter after 2 hours of hypoxia was suppressed significantly by treatment with anti-IL-6 antibody. $\mathbf{b}$, The chemiluminescence was also significantly higher in group $2 \mathrm{H}$ than in group $\mathrm{N}$. The increase in this parameter after 2 hours of hypoxia was suppressed significantly by treatment with anti-IL- 6 antibody.

dial injury. These findings from the present study and previous studies endorsed our hypothesis that IL-6, produced in ischemic cardiac myocytes, leads to enhancement of neutrophil-mediated reperfusion injury of the myocardium.

The mechanisms of the neutrophil-mediated reperfusion injury resemble, at first glance, the mechanisms of host defense against foreign matter invasion by neutrophil migration in the presence of inflammation. ${ }^{3}$ However, our findings suggest that (a) Endothelial cells

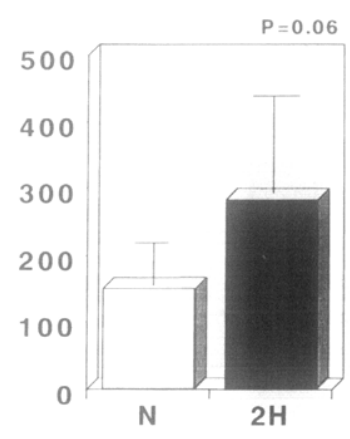

(b) Myocytes

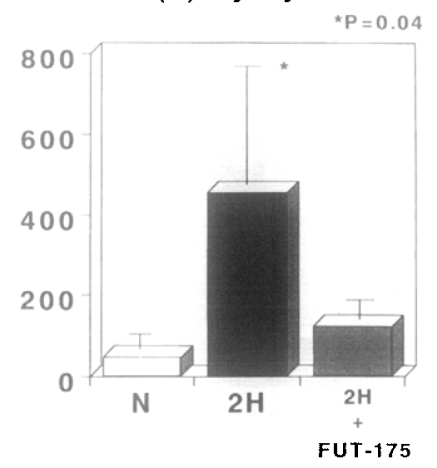

Fig. 6. IL-6 production by myocytes and endothelial cells. a, IL-6 production by coronary endothelial cells did not differ significantly between group $2 \mathrm{H}$ and group $\mathrm{N}$. b, The IL-6 level in the supernatant of myocyte cultures was significantly higher in group $2 \mathrm{H}$ than in group N. IL-6 production increased significantly with time. Treatment with FUT-175 significantly suppressed IL-6 production by myocytes.

neutrophil migration into ischemic myocardium causes exacerbation of myocardial damage rather than repair. Measures to prevent neutrophil migration, therefore, seem essential in achieving better protection of the myocardium. Development of a clinically applicable anti-IL-6 antibody, as well as anti-intercellular adhesion molecule- 1 and antiCD18 antibodies will therefore be useful in preventing neutrophil-mediated reperfusion injury during active thrombolytic therapy for myocardial infarction and during cardiac operations. Moreover, blood or coronary sinus IL-6 levels seem to be significant as indicators of ischemic injury and myocardial infarction.

\section{Conclusion}

1. During cardiac operations on adult human beings, myocardial IL-6 production increased significantly after reperfusion, and this increase was suppressed by FUT-175, a protease inhibitor.

2. When a coincubation of myocytes and endothelial cells was cultured in hypoxic conditions for 2 hours, neutrophil migration increased significantly, accompanied by a significant increase in the proportion of damaged myocytes. These changes were suppressed significantly by an anti-IL- 6 monoclonal antibody.

3. Exposure of myocytes to hypoxia for 2 hours resulted in a significant increase in IL-6 production as compared with hypoxic endothelial cells. This change was suppressed by a protease inhibitor. 
These results suggest that during cardiac operations, IL- 6 production by myocardium (especially myocytes) increases in the presence of ischemia and that the IL-6 thus produced enhances neutrophilmediated reperfusion injury in myocardium.

\section{REFERENCES}

1. Engler RT, Schmid-Schoenbein GW, Pavelec RS. Leukocyte capillary plugging in myocardial ischemia and reperfusion in the dog. Am J Pathol 1983;111:98-111.

2. Entman ML, Youker K, Shappel SB, Siegel C, Rothlein R, Dreyer WJ, et al. Neutrophil adherence to isolated adult canine myocytes: evidence for a CD18-dependent mechanism. J Clin Invest 1991;88:1216-23.

3. Entman ML, Michael LH, Rossen RD, Dreyer WJ, Anderson DC, Taylor AA, et al. Inflammation in the course of early myocardial ischemia. FASEB J 1991;5:2529-37.

4. Sawa Y, Nakano S, Shimazaki Y, Nishimura M, Kuratani T, Matsuda $\mathrm{H}$. Myocardial protective effect and its mechanism of leukocyte-depleted reperfusion in neonatal rabbit hearts. Ann Thorac Surg 1994;58:1386-91.

5. Sawa Y, Matsuda H, Shimazaki Y, Kaneko M, Nishimura M, Amemiya A, et al. Evaluation of leukocyte-depleted terminal blood cardioplegic solution in patients undergoing elective and emergency coronary artery bypass grafting. J Thorac Cardiovase Surg 1994;108:1125-31.

6. Sawa Y, Taniguchi K, Kadoba K, Nishimura M, Ichikawa H, Amemiya $A$, et al. Leukocyte depletion attenuates reperfusion injury in patients with left ventricular hypertrophy. Circulation 1996;93:1640-6.

7. Chen CC, Matsuda H, Sawa Y. Effect of acyclic adenosine monophosphate phosphodiesterase inhibitor, DN-9693, on myocardial reperfusion injury. Ann Thorac Surg 1991;52: 495-9.

8. Faes FC, Sawa $\mathrm{Y}$, Ichikawa $\mathrm{H}$, Shimazaki $\mathrm{Y}$, Ohashi $\mathrm{T}$, Fukuda $\mathrm{H}$, et al. Inhibition of $\mathrm{Na} / \mathrm{H}$ exchanger attenuates neutrophil-mediated reperfusion injury. Ann Thorac Surg 1995:60:377-81.

9. Sawa Y, Bernotat-Danieloski S, Stoll M, Schaper J. CD18-
ICAM-1 dependent neutrophil-endothelial cell and neutrophil-myocyte adherence is promoted by hypoxic myocyte: evidence for hypoxic myocyte derived activating factors. Circulation 1991;84(Suppl):II85.

10. Ikeda U, Ohkawa F, Seino Y, Yamamoto K, Hidaka Y, Kasahara $T$, et al. Serum interleukin- 6 levels become clevated in acute myocardial infarction. $\mathrm{J}$ Mol Cell Cardiol 1992;24:579-84

11. Miyao Y, Yasue H, Ogawa H, Misumi I, Masuda T Sakamoto $\mathrm{T}$, et al. Elevated plasma interleukin- 6 levels in patients with acute myocardial infarction. Am Heart J 1993;126:1299-304.

12. Herskowitz A, Choi S, Ansari AA, Wesseling S. Cytokine mRNA expression in post ischemic/reperfused myocardium. Am J Pathol 1995;146:419-28.

13. Yamauchi-Takihara K, Ihara Y, Ogata A, Yoshizaki K, Azuma J, Kishimoto T. Hypoxic stress induces cardiac myocyte-derived interleukin-6. Circulation 1995;91:1520-4.

14. Sawa Y, Shimazaki Y, Kadoba K, Masai T, Fukuda H, Ohata $\mathrm{T}$, et al. Attenuation of cardiopulmonary bypass-derived inflammatory reactions reduces myocardial reperfusion injury in cardiac operations. J Thorac Cardiovasc Surg 1996: 111:29-35

15. Rugo HS, O’Hanley P, Bishop AG, Pearce MK, Abrams JS, Howard $\mathrm{M}$, et al. Local cytokine production in a murine model of Escherichia coli pyelonephritis. J Clin Invest 1992; 89:1032-9.

16. Chantry D, Turner M, Abney $\mathbf{E}$, Feldmann M. Modulation of cytokine production by transforming growth factor- $\beta_{1} . \mathrm{J} \mathrm{Im}$ munol 1989;142:4295-4300.

17. Youker K, Smith CW, Anderson DC, Miller D, Michael LH, Rossen RD, et al. Neutrophil adherence to isolated adult cardiac myocytes: induction by cardiac lymph collected during ischemia and reperfusion. J Clin Invest 1992;89:602-9.

18. Kukielka GL, Smith CW, LaRosa GJ, Manning AM, Mendoza LH, Daly TJ, et al. Interleukin-8 gene induction in the myocardium after ischemia and reperfusion in vivo. $\mathrm{J}$ Clin Invest 1995;95:89-103

19. Finkel MS, Oddis CV, Jacob TD, Watkins SC, Hattler BG, Simmons RL. Negative inotropic effects of cytokines on the heart mediated by nitric oxide. Science 1992;257:387-9. 